\title{
Purification of Human Very-Long-Chain Acyl-Coenzyme A Dehydrogenase and Characterization of Its Deficiency in Seven Patients
}

\author{
Toshifumi Aoyama, * Masayoshi Souri, * Seiichi Ushikubo, ${ }^{\ddagger}$ Takehiko Kamijo, ${ }^{\ddagger}$ Seiji Yamaguchi, ${ }^{5}$ Richard I. Kelley," \\ William J. Rhead, ' Kimiaki Uetake, ** Kay Tanaka, \# and Takashi Hashimoto* \\ ${ }^{*}$ Department of Biochemistry, Shinshu University School of Medicine, Matsumoto 390, Japan; ${ }^{\ddagger}$ Department of Pediatrics, Shinshu \\ University School of Medicine, Matsumoto 390, Japan; ${ }^{5}$ Department of Pediatrics, Shimane Medical University, Izumo 693, Japan; \\ "Department of Pediatrics, Johns Hopkins University School of Medicine, Baltimore, Maryland 21205; 'Department of Pediatrics, \\ University of Iowa, Iowa City, Iowa 52242; **Department of Pediatrics, Hokkaido University School of Medicine, Sapporo 060, Japan; \\ and ${ }^{\ddagger \ddagger}$ Department of Genetics, Yale University School of Medicine, New Haven, Connecticut 06510
}

\section{Abstract}

Mitochondrial very-long-chain acyl-coenzyme A dehydrogenase (VLCAD) was purified from human liver. The molecular masses of the native enzyme and the subunit were estimated to be 154 and $70 \mathrm{kD}$, respectively. The enzyme was found to catalyze the major part of mitochondrial palmitoylcoenzyme A dehydrogenation in liver, heart, skeletal muscle, and skin fibroblasts (89-97, 86-99, 96-99, and 78-87\%, respectively).

Skin fibroblasts from 26 patients suspected of having a disorder of mitochondrial $\beta$-oxidation were analyzed for VLCAD protein using immunoblotting, and 7 of them contained undetectable or trace levels of the enzyme. The seven deficient fibroblast lines were characterized by measuring acyl-coenzyme A dehydrogenation activities, overall palmitic acid oxidation, and VLCAD protein synthesis using pulse-chase, further confirming the diagnosis of VLCAD deficiency. These results suggested the heterogenous nature of the mutations causing the deficiency in the seven patients.

Clinically, all patients with VLCAD deficiency exhibited cardiac disease. At least four of them presented with hypertrophic cardiomyopathy. This frequency $(>57 \%)$ was much higher than that observed in patients with other disorders of mitochondrial long-chain fatty acid oxidation that may be accompanied by cardiac disease in infants. (J. Clin. Invest. 1995. 95:2465-2473.) Key words: fatty acid $\beta$-oxidation - pulse-chase experiment - palmitoyl-coenzyme A • mitochondria • hypertrophic cardiomyopathy

\section{Introduction}

Inborn errors of fatty acid metabolism are a group of diseases affecting infants and children. More than 13 separate inherited disorders of mitochondrial fatty acid $\beta$-oxidation have been described in humans, and the characterization of these disorders at the clinical and biochemical levels has progressed (1-4).

Address correspondence to Dr. Toshifumi Aoyama, Department of Biochemistry, Shinshu University, Matsumoto 390, Japan. Phone: 263-354600x5181; FAX: 263-33-6458.

Received for publication 23 September 1994 and in revised form 21 February 1995.

J. Clin. Invest.

(c) The American Society for Clinical Investigation, Inc. $0021-9738 / 95 / 06 / 2465 / 09 \quad \$ 2.00$

Volume 95, June 1995, 2465-2473
Within the group of acyl-coenzyme A ( CoA) dehydrogenases that catalyze the first reaction in the mitochondrial $\beta$-oxidation of straight-chain fatty acid of various chain lengths, deficiencies of three enzymes have been reported (5-18). Among these defects, medium-chain acyl-CoA dehydrogenase (MCAD) ${ }^{1}$ deficiency is relatively common. MCAD deficiency often remains undetected and asymptomatic for months or years until a metabolic crisis is induced by infection or fasting. In contrast to MCAD deficiency, other defects, such as short-chain acyl-CoA dehydrogenase and long-chain acyl-CoA dehydrogenase (LCAD) deficiencies, are rare. The detailed clinical descriptions of these defects are quite variable, and many patients die before $1 \mathrm{yr}$ of age (12-20).

Recently, a novel fatty acyl-CoA dehydrogenase, "verylong-chain acyl-CoA dehydrogenase (VLCAD)," has been purified from rat liver mitochondria and characterized in our laboratory (21). VLCAD is unique among acyl-CoA dehydrogenases in its size, structure, and intramitochondrial distribution. While the other acyl-CoA dehydrogenases are homotetramers of a 43-45-kD subunit, VLCAD is a dimer of a 70-kD subunit, each containing a $29-\mathrm{kD}$ extra polypeptide compared with the subunits of other dehydrogenases. VLCAD also differs from three other acyl-CoA dehydrogenases in that it is loosely bound to the mitochondrial inner membrane and requires detergent for solubilization, while three others are readily extractable into the soluble fraction without detergent, indicating that they are localized in the mitochondrial matrix. It is noteworthy that, catalytically, VLCAD has a 10 times higher specific activity toward palmitoyl-CoA than that of LCAD. It was considered previously that LCAD was mainly responsible for the dehydrogenation of palmitoyl-CoA. In addition, VLCAD is active toward $\mathrm{CoA}$ esters of longer chain substrates such as arachidoylCoA, behenoyl-CoA, and lignoceroyl-CoA, but it is not active toward those of medium- and short-chain substrates (21).

To search for patients with VLCAD deficiency, we previously determined palmitoyl-CoA dehydrogenase activity in skin fibroblasts from seven patients who were suspected of having a disorder of mitochondrial $\beta$-oxidation. Among them, we found abnormally low VLCAD activity in two patients (22). At the same time, skin fibroblasts from these two patients were found to have trace amounts of VLCAD protein in immunoblot analysis. Furthermore, three additional VLCAD patients were

1. Abbreviations used in this paper: FAD, flavine adenine dinucleotide; LCAD, long-chain acyl-CoA dehydrogenase; MCAD, medium-chain acyl-CoA dehydrogenase; POCA, sodium 2-[5-(4-chlorophenyl)pentyl]-oxirane-2-carboxylate; VLCAD, very-long-chain acyl-CoA dehydrogenase. 
identified among those who were diagnosed previously as having LCAD deficiency (23).

In this paper, we describe the purification and characterization of VLCAD from normal human liver and report in detail our biochemical and immunochemical investigations of fibroblasts from these five VLCAD-deficient patients and two additional patients who were subsequently identified.

\section{Methods}

\section{Case history}

The case histories were partially described in the previous reports (12, $13,22,24-26)$. We describe them as follows. Patient 1 was first noted to be hypotonic and developmentally delayed at age $2 \mathrm{mo}$. He then presented acutely at the age of 3 mo with hypoketotic hypoglycemia, hepatocellular dysfunction, and cardiomyopathy. Initial laboratory examinations showed abnormally increased blood levels of bilirubin, GOT, GPT, uric acid, and lactic acid, as well as a low level of total serum carnitine and increased levels of long-chain acylcarnitine esters. Urinary organic acid analysis showed a medium-chain 3-hydroxydicarboxylic aciduria without increased levels of medium-chain acylglycines. Despite some clinical improvement of the patient after restriction to a high carbohydrate, low-fat diet, the cardiac disease worsened and he died after 3 wk of hospitalization. At autopsy, severe hepatocellular injury and lipid storage in many tissues were found. Patient 2 presented at the age of 4 mo with nystagmus, hepatomegaly, poor head control, generalized hypotonia, myoclonic jerks, hypoglycemia, and cardiomyopathy. Laboratory examinations revealed mild metabolic acidosis, abnormal liver function tests, and hyperammonemia. Urinary organic acid analysis revealed lactic, glutaric, adipic, octanedioic, decanedioic, and palmitic acid excretion. Subsequently, the muscular hypotonia worsened and she developed significant cardiomyopathy and hypoglycemia. She died on day 19 of hospitalization after cardiac transplantation. Microscopic examination of autopsy tissues revealed severe macrovesicular lipid storage in heart, liver, kidneys, and skeletal muscle. Patients 3,4 , and 5 were initially reported as having $\operatorname{LCAD}$ deficiency $(12,13,25,26)$, but later, were shown to be deficient in VLCAD, rather than in LCAD, using immunoblot analysis (23). Patient 6 was reported previously to have LCAD deficiency $(12,13)$, but has been found to be deficient in VLCAD in this study. Patient 7, a 6-yr-old Japanese girl, is the first child of healthy unrelated young parents. She presented at age $24 \mathrm{~h}$ with intermittent respiratory distress, premature ventricular contraction of the heart, and hepatomegaly. Laboratory data disclosed hypoglycemia and mild liver dysfunction (GOT 103 IU/liter, GPT 12 IU/liter). Her general condition, metabolic acidosis, and arrhythmia were gradually improved by intravenous glucose infusion for $4 \mathrm{~d}$. She presented again at the age of 17 mo with hepatomegaly and severe muscle hypotonia. She had not walked alone for the last 3 wk. Laboratory data on admission disclosed metabolic acidosis, mild liver dysfunction, and dicarboxylic aciduria. Muscle biopsy revealed abnormal accumulation of lipid droplets. After the two serious episodes in the infantile period, she had repeated hospital admissions several times a year for persistent emesis with nonketotic hypoglycemia, metabolic acidosis, elevated creatine kinase levels, and organic aciduria, which were improved by intravenous glucose infusion for 3-4 d. Clinical features of the seven patients are summarized in Table I.

\section{Purification of human VLCAD}

All procedures except the heat step were carried out at $4^{\circ} \mathrm{C}$.

Step 1: extraction. Frozen normal human liver sample ( $100 \mathrm{~g})$ obtained at autopsy was homogenized with $400 \mathrm{ml}$ of $10 \mathrm{mM} \mathrm{K}_{2} \mathrm{HPO}_{4}$ containing $5 \mathrm{mM}$ 2-mercaptoethanol, $1 \mathrm{mM}$ benzamidine, and $0.5 \mathrm{mM}$ phenylmethylsulfonyl fluoride in a blender. The homogenate was centrifuged at $1,000 \mathrm{~g}$ for $10 \mathrm{~min}$, and the precipitate was discarded. The supernatant was centrifuged at $100,000 \mathrm{~g}$ for $60 \mathrm{~min}$. The pellet was

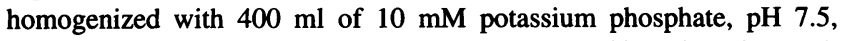
containing $1 \%(\mathrm{wt} / \mathrm{vol}$ ) sodium cholate, $0.5 \mathrm{M} \mathrm{NaCl}$, and a mixture $\mathrm{A}$ composed of $0.1 \%$ (wt/vol) hexamethylphosphoric triamide, $2 \mathrm{mM} 2$ mercaptoethanol, $1 \mathrm{mM}$ EDTA, and $10 \mu \mathrm{M}$ flavine adenine dinucleotide (FAD). The suspension was centrifuged at $100,000 \mathrm{~g}$ for $60 \mathrm{~min}$.

Step 2: heat and ammonium sulfate fractionation. The extract was heated $\left(52-53^{\circ} \mathrm{C}\right)$ and then fractionated with ammonium sulfate as described previously (21).

Step 3: phosphocellulose column chromatography. The desalted enzyme solution was applied to a phosphocellulose column $(1.5 \times 12 \mathrm{~cm})$ equilibrated with $10 \mathrm{mM}$ potassium phosphate, $\mathrm{pH} 7.5$, containing $0.2 \%$ (wt/vol) Tween 20 and mixture A. The column was washed with the same buffer, and the elution was performed with a linear gradient of $10-200 \mathrm{mM}$ potassium phosphate, $\mathrm{pH} 7.5$, containing $0.2 \%$ (wt/vol) Tween 20 and mixture $A$, in a total volume of $120 \mathrm{ml}$.

Step 4: DEAE-cellulose column chromatography. Active fractions were pooled and diluted threefold with $0.2 \%$ (wt/vol) Tween 20 containing mixture A and applied to a DEAE-cellulose column $(1.5 \times 8$ $\mathrm{cm}$ ) equilibrated with $30 \mathrm{mM}$ potassium phosphate, $\mathrm{pH} 7.5$, containing $0.2 \%$ (wt/vol) Tween 20 and mixture A. The column was washed with the same buffer, and the elution was performed with a linear gradient of $30-300 \mathrm{mM}$ potassium phosphate, $\mathrm{pH} 7.5$, containing $0.2 \%$ (wt/ vol) Tween 20 and mixture $A$, in a total volume of $80 \mathrm{ml}$.

Step 5: Sepharose $6 B$ column chromatography. Active fractions were pooled and concentrated by Ultracent-30 (TOSO, Tokyo, Japan). The concentrated sample was applied to a Sepharose 6B column (1.2 $\times 48 \mathrm{~cm}$ ) equilibrated with $50 \mathrm{mM}$ potassium phosphate, $\mathrm{pH} 7.5$, containing $0.2 \%$ (wt/vol) Tween $20,0.2 \mathrm{M} \mathrm{NaCl}$, and mixture A. The same buffer was used for the elution. Active fractions were pooled and concentrated by $50 \%$ polyethylene glycol No. 6000 in a thin dialysis tube (cellulose tube, 18/32 inch). The concentrated sample was dia-

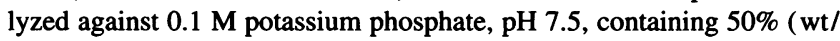
vol) glycerol, and stored under $-20^{\circ} \mathrm{C}$.

\section{Western blot analysis}

Sodium dodecyl sulfate (SDS)-polyacrylamide gel electrophoresis and Western blotting were performed by the described method (27), using $10 \%$ acrylamide, $0.3 \%$ bisacrylamide-containing SDS-polyacrylamide gels. Nitrocellulose sheet was incubated with phosphate-buffered saline containing anti-human VLCAD antibody. Protein detection was achieved using alkaline phosphatase-conjugated goat anti-rabbit IgG antibody (KPL Laboratories, Gaithersburg, MD) and nitro-blue-tetrazolium/5-bromo-4-chloro-3-indolyl phosphate substrate kit (Pierce, Rockford, IL).

\section{Pulse-Chase experiment}

Fibroblasts were grown in a $28-\mathrm{cm}^{2}$ dish for pulse-chase experiments. The cells were washed twice with methionine-free modified Eagle's medium containing $5 \%(\mathrm{vol} / \mathrm{vol})$ dialyzed fetal calf serum and then incubated for $1 \mathrm{~h}$ in the same medium at $37^{\circ} \mathrm{C}$. The medium was replaced with $1 \mathrm{ml}$ of the same medium containing $120 \mu \mathrm{Ci}$ of $\left[{ }^{35} \mathrm{~S}\right.$ ] methionine (New England Nuclear, Wilmington, DE). After a 1.5-h incubation, the labeling medium was changed to the standard medium, and the preparation was chased for 6,24 , or $48 \mathrm{~h}$, respectively. The labeled cells were washed twice with phosphate-buffered saline and harvested in $500 \mu \mathrm{l}$ of $10 \mathrm{mM}$ Tris-Cl (pH 7.5)/2 mM EDTA $/ 0.1 \%$ SDS $/ 0.1 \%$ Triton X$100 / 0.1 \%$ bovine serum albumin $/ 10 \mathrm{mM}$ methionine $/ 0.02 \%$ sodium azide/protease inhibitors. The suspension was sonicated and centrifuged at $10,000 \mathrm{~g}$ for $10 \mathrm{~min}$, and the supernatant was preserved for immunoprecipitation. ${ }^{35} \mathrm{~S}$-Labeled proteins in the fibroblast extracts were immunoprecipitated with anti-human VLCAD antibody and $50 \mu \mathrm{l}$ of $20 \%$ (wt/vol) of fixed Staphylococcus aureus cells. The recovered labeled proteins were subjected to SDS-polyacrylamide gel electrophoresis and fluorography. Competition experiments were done using an excess amount of the purified human enzymes during the immunoprecipitation.

\section{Long-chain fatty acid oxidation}

Fibroblasts were seeded in $8-\mathrm{cm}^{2}$ dishes $24 \mathrm{~h}$ before experiments. The medium was changed to modified Eagle's medium with or without $20 \mu \mathrm{M}$ sodium 2-[5-(4-chlorophenyl)pentyl]-oxirane-2-carboxylate 


\begin{tabular}{|c|c|c|c|c|c|c|c|}
\hline Patient & 1 & 2 & 3 & 4 & 5 & 6 & 7 \\
\hline Prior code & Patient 1 & Patient 2 & R-1 & A-1 & Patient 2 & Patient 3 & - \\
\hline Reference & 22 & 22,24 & 12,23 & 12,23 & 25 & 25 & - \\
\hline Sex & $\mathbf{M}$ & $\mathbf{F}$ & $\mathbf{M}$ & $\mathbf{M}$ & $\mathbf{M}$ & $\mathbf{M}$ & $\mathrm{F}$ \\
\hline \multicolumn{8}{|l|}{ Initial episode } \\
\hline Age of first presentation & $2 \mathrm{mo}$ & $4 \mathrm{mo}$ & $1.5 \mathrm{~d}$ & $4 \mathrm{mo}$ & $12 \mathrm{~d}$ & $2 \mathrm{mo}$ & $1 \mathrm{~d}$ \\
\hline Fasting coma & + & + & + & + & + & + & + \\
\hline Hepatic disease & + & + & + & + & + & + & + \\
\hline \multirow[t]{2}{*}{ Cardiac disease } & + & + & + & + & + & + & + \\
\hline & Cardiomyopathy & $\begin{array}{c}\text { Cardiomyopathy, } \\
\text { cardiomegaly }\end{array}$ & $\begin{array}{c}\text { Cardiomyopathy, } \\
\text { cardiomegaly }\end{array}$ & $\begin{array}{c}\text { Cardiomyopathy, } \\
\text { cardiomegaly }\end{array}$ & & & Arrhythmia \\
\hline Muscle weakness & + & $?$ & + & $?$ & + & $?$ & + \\
\hline Age of death & $4 \mathrm{mo}$ & $5 \mathrm{mo}$ & - & $6.5 \mathrm{mo}$ & $14 d$ & $2.5 \mathrm{mo}$ & - \\
\hline
\end{tabular}

(POCA) (Byk Gulden Pharmazeutika, Konstanz, Germany), and the preparation was incubated for $3 \mathrm{~h}$ at $37^{\circ} \mathrm{C}$. The fibroblasts were further incubated for $1 \mathrm{~h}$ using fetal calf serum-free medium at the same concentration of POCA to exclude cold fatty acids from cells. Fatty acid oxidation reaction was initiated by adding $4 \mathrm{nmol}\left[1-{ }^{14} \mathrm{C}\right]$ palmitic acid ( $54 \mathrm{mCi} / \mathrm{mmol}$; American Radiolabeled Chemicals, St. Louis, MO) dissolved in $10 \mathrm{mM} \alpha$-cyclodextrin to $1 \mathrm{ml}$ of fresh fetal calf serumfree medium, with or without POCA. The reaction was run for $1 \mathrm{~h}$ and stopped by adding $0.15 \mathrm{ml}$ of $10 \%$ bovine serum albumin and $0.2 \mathrm{ml}$ of $3 \mathrm{~N}$ perchloric acid. The contents in a dish were transferred into a $15-\mathrm{ml}$ tube and centrifuged at $2,000 \mathrm{~g}$ for $10 \mathrm{~min}$. The unreacted fatty acid in the supernatant was removed with $5 \mathrm{ml}$ of $n$-hexane, with three extractions. Radioactive degradation products in the water phase were counted $(28,29)$.

\section{Other methods}

Protein concentration was determined using a $\mathrm{BCA}^{2}$ protein determination kit (Pierce). Acyl-CoA dehydrogenation activities in liver, heart, and skeletal muscle were measured by the method as described (30). The activities in fibroblasts were measured by the method coupling with ferricenium ion (31). All samples were treated with Triton X-100 as described (22).

\section{Results}

Purification of human VLCAD. The purification of human liver mitochondrial VLCAD is summarized in Table II. The yield was high, even though whole liver homogenate was used as the starting material.

Table II. Summary of the Purification of VLCAD

\begin{tabular}{lccccc}
\hline \multicolumn{1}{c}{ Step } & $\begin{array}{c}\text { Total } \\
\text { activity }\end{array}$ & $\begin{array}{c}\text { Total } \\
\text { protein }\end{array}$ & $\begin{array}{c}\text { Specific } \\
\text { activity }\end{array}$ & Yield & $\begin{array}{c}\text { Purification } \\
\text { factor }\end{array}$ \\
\hline units & $m g$ & unit/mg & $\%$ & fold \\
Crude extract & 29.4 & 1533 & 0.019 & 100 & 1 \\
Heat treatment & 21.0 & 936 & 0.022 & 71.4 & 1.2 \\
Ammonium sulfate & 15.1 & 225 & 0.067 & 51.4 & 3.5 \\
Phosphocellulose & 12.6 & 11.7 & 1.08 & 42.9 & 57 \\
DEAE-cellulose & 9.4 & 3.4 & 2.76 & 32.0 & 145 \\
Sepharose 6B & 8.6 & 1.4 & 6.14 & 29.2 & 323 \\
& & & & & \\
\hline
\end{tabular}

The homogenate obtained from $100 \mathrm{~g}$ of human liver was used.
The results of SDS-polyacrylamide gel electrophoresis of the final preparation are shown in Fig. 1. Purified human VLCAD migrated slightly faster than rat VLCAD (21). The molecular mass of the subunit of human VLCAD was estimated to be $70 \mathrm{kD}$. The native molecular mass of human VLCAD was estimated by gel chromatography using Ultrogel AcA 34 and Sephadex G-150 (TOSO, Tokyo, Japan). The enzyme activity peak corresponded to a molecular mass of $154 \mathrm{kD}$ (data not shown). Thus, human VLCAD is probably a homodimer, as is the case for rat VLCAD (21).

The final preparation was used for FAD determination. The protein was deproteinized with trichloroacetic acid, and the supernatant was neutralized with $\mathrm{K}_{2} \mathrm{HPO}_{4}$. The absorbance of this solution at $450 \mathrm{~nm}$ was measured. Based on the molar extinction coefficient of FAD at this wavelength $\left(11,300 \mathrm{M}^{-1} \mathrm{~cm}^{-1}\right)(32)$,

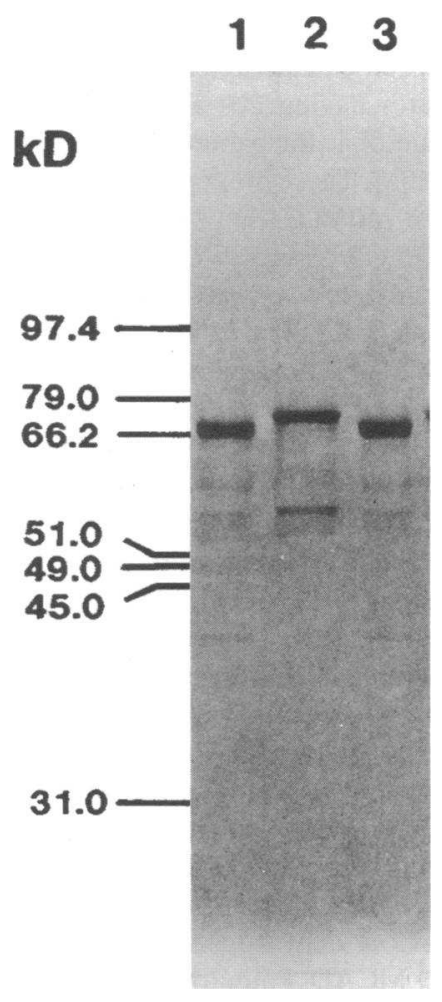

Figure 1. SDS-polyacrylamide gel electrophoresis of purified preparations. The enzymes were analyzed on a $10 \%$ gel. Lanes 1 and 3 , purified human VLCAD $(2 \mu \mathrm{g})$; lane 2 , purified rat VLCAD $(2 \mu \mathrm{g})$. The protein band was detected with Coomassie brilliant blue R-250. 
Table III. Acyl-CoA Dehydrogenase Activity in Human Liver and Heart

\begin{tabular}{|c|c|c|c|c|}
\hline \multirow[b]{2}{*}{ Sample } & \multicolumn{2}{|c|}{ Palmitoyl-CoA dehydrogenation } & \multicolumn{2}{|c|}{ Octanoyl-CoA dehydrogenation } \\
\hline & -Antibody & +Anti-VLCAD & -Antibody & + Anti-MCAD \\
\hline \multicolumn{5}{|l|}{ Liver } \\
\hline 1 & 5.90 & 0.19 & 3.38 & 0.62 \\
\hline 2 & 5.05 & 0.30 & 2.20 & 0.33 \\
\hline 3 & 6.47 & 0.60 & 2.79 & 0.34 \\
\hline 4 & 6.33 & 0.68 & 3.17 & 0.51 \\
\hline 5 & 5.42 & 0.27 & 2.88 & 0.30 \\
\hline \multicolumn{5}{|l|}{ Heart } \\
\hline 1 & 5.80 & 0.83 & 3.03 & 0.31 \\
\hline 2 & 15.45 & 0.17 & 4.04 & 0.53 \\
\hline 3 & 17.97 & 0.24 & 4.31 & 0.46 \\
\hline
\end{tabular}

About $2 \mathrm{mg}$ of liver (five samples) or heart (three samples) was suspended in $200 \mu$ l of solution containing $67 \mathrm{mM}$ potassium phosphate (pH 7.5), $200 \mathrm{mM}$ sodium chloride, and $0.6 \%$ (wt/vol) Triton X-100. The suspension was sonicated, and the solution was centrifuged at 3,000 $g$ for $5 \mathrm{~min}$. $60 \mu \mathrm{l}$ of the supernatant fraction was incubated with either $108 \mu \mathrm{g}$ of anti-VLCAD IgG or $120 \mu \mathrm{g}$ of anti-MCAD IgG and $120 \mu \mathrm{g}$ of bovine serum albumin, respectively, for $30 \mathrm{~min}$ on ice. The amount of added IgG corresponded to approximately five times as much as it gave strong reduction to the reaction of human acyl-CoA dehydrogenase in livers. The mixtures were then centrifuged at $4,000 \mathrm{~g}$ for $12 \mathrm{~min}$. The supernatant fraction was used for the assays. Activity unit is milliUnits per milligram of protein. Values are means of three or four assays.

the content of FAD was calculated to be $0.92 \mathrm{~mol} / \mathrm{mol}$ of the subunit of the enzyme.

As shown in Table $I$, the final preparation had a high specific activity (6.14 unit $[\mu \mathrm{mol} / \mathrm{min}] / \mathrm{mg})$ toward palmitoyl-CoA as a substrate. It was inactive toward acyl-CoAs with shorter than eight-carbon chain lengths. A very low activity was found with substrates having 10- and 12-carbon chain lengths. It exhibited significant activity with substrates having 20- and 24-carbon chain lengths. Apparent $K_{\mathrm{m}}$ value of it for palmitoyl-CoA and phenazine methosulfate was $1.1 \mathrm{mM}$. These properties of human VLCAD were similar to those of rat VLCAD (21).

Contribution of human VLCAD to palmitoyl-CoA and octanoyl-CoA dehydrogenation activities in liver and heart. Palmitoyl-CoA dehydrogenation is considered to be catalyzed by four enzymes, i.e., MCAD, LCAD, VLCAD, and peroxisomal acylCoA oxidase in rat liver (33). The contribution of VLCAD to the reaction in normal human liver and heart was measured using anti-human VLCAD antibody. As shown in Table III, the major part (89-97\% in liver, $86-99 \%$ in heart) of the mitochondrial palmitoyl-CoA dehydrogenase activity was catalyzed by VLCAD. Contribution of VLCAD to the reaction in normal human skeletal muscle was also examined. The activities without and with anti-human VLCAD antibody were $1.36 \pm 0.12$ and $0.04 \pm 0.03 \mathrm{mU} / \mathrm{mg}$ protein $(n=3)$, respectively. These data indicate that the major part $(>97 \%)$ of the activity in skeletal muscle was catalyzed by VLCAD as well as in liver and heart, and VLCAD content in skeletal muscle was much lower than that in liver and heart, as described in rat (29). On the other hand, the major part (82-90\% in liver, $87-$ $90 \%$ in heart) of the octanoyl-CoA dehydrogenation activity by the enzymes was catalyzed by MCAD. Palmitoyl-CoA dehy- drogenation activities in human livers in the absence and presence of anti-rat LCAD antibody were $5.83 \pm 0.69$ and $5.66 \pm 0.91 \mathrm{mU} / \mathrm{mg}$ protein $(n=5)$, respectively. OctanoylCoA dehydrogenation activities were $2.88 \pm 0.38$ and $2.42 \pm 0.44$ $\mathrm{mU} / \mathrm{mg}$ protein $(n=5)$, respectively. Contributions of LCAD to the palmitoyl-CoA and octanoyl-CoA dehydrogenation activities in liver were then $<3$ and $16 \%$, respectively. These and other previous data (34) suggest that the role of LCAD is limited the oxidation of $\mathrm{C}_{10}-\mathrm{C}_{14}$ acyl-CoAs. To further study the role of VLCAD in palmitoyl-CoA oxidation, five human liver samples and three heart samples described in Table III were examined by immunoblot analysis. As shown in Fig. 2 A, a clear single band of VLCAD was detected in each of the livers. Specific activity ranged from 4.9 to $6.0 \mathrm{unit} / \mathrm{mg}$, the values similar to that ( $6.14 \mathrm{unit} / \mathrm{mg})$ of purified human VLCAD (Table II). In Fig. $2 B$, a clear band of VLCAD was also found in each of the hearts. Specific activity ranged from 6.2 to 8.3 unit/mg, values somewhat higher than that $(6.14 \mathrm{unit} / \mathrm{mg})$ of purified human VLCAD. These data support the notion that VLCAD is the major catalyst for mitochondrial palmitoyl-CoA dehydrogenation both in liver and heart. Fig. 2 also indicates that anti-human VLCAD antibody used in this study did not cross-react with MCAD and LCAD (42 kD).

Immunoblot analysis of skin fibroblasts. Using immunoblotting, a clear band of the VLCAD protein was found in the control fibroblasts. The VLCAD content in the control fibroblasts $(n=6)$ was estimated to be $0.15-0.24 \mu \mathrm{g} / \mathrm{mg}$ protein in a separate experiment. Skin fibroblasts from 26 candidates suspected of having a disorder of mitochondrial $\beta$-oxidation were then analyzed for the VLCAD protein. The VLCAD protein was not detected at all in six samples (patients 1-6), and only a trace amount of the VLCAD protein was found in another (patient 7) (Fig. 3). Several bands of varying intensities, migrating at higher rates than the VLCAD protein, were detected in all of the seven samples (patients 1-7). These bands may be intermediates on the degradation pathway in the fibroblasts concerning the synthesized VLCAD, because the bands had specific cross-reactivity against anti-human VLCAD antibody. Similar bands were observed in rat hepatoma cells expressing rat VLCAD (29). In each of the cells from the 19 other candidates, the amount of VLCAD protein that was similar to those found in the control fibroblasts was detected (data not shown). We also examined other mitochondrial proteins and a peroxisomal protein using immunoblot analysis. These include: shortchain acyl-CoA dehydrogenase, MCAD, LCAD, enoyl-CoA hydratase/3-hydroxyacyl-CoA dehydrogenase/3-ketoacyl-CoA thiolase trifunctional protein $(35,36)$, and acyl-CoA oxidase. The intensities of the immunoblot signals for these proteins in the seven VLCAD-deficient patients' fibroblasts were essentially the same as those for the controls (data not shown). These results indicate that the patients' fibroblasts were deficient only for the VLCAD protein.

Pulse-Chase experiment. The seven patients' fibroblasts and control fibroblasts were incubated for $1.5 \mathrm{~h}$ in the medium containing $\left[{ }^{35} \mathrm{~S}\right]$ methionine and then incubated for 6,24 , or 48 $h$, respectively, with the standard medium. The fluorographic bands at the 1.5-h pulse, and 6-, 24-, and 48-h chases were clearly observed in the control fibroblasts, and the relative intensity at each time point was $100,74,33$, and 18 , respectively (Fig. $4 A$ ). In contrast, a very faint fluorographic band was observed only at the 1.5 -h pulse in patient 1 's fibroblasts (Fig. 4A). The band seemed to migrate at the same rate as that 


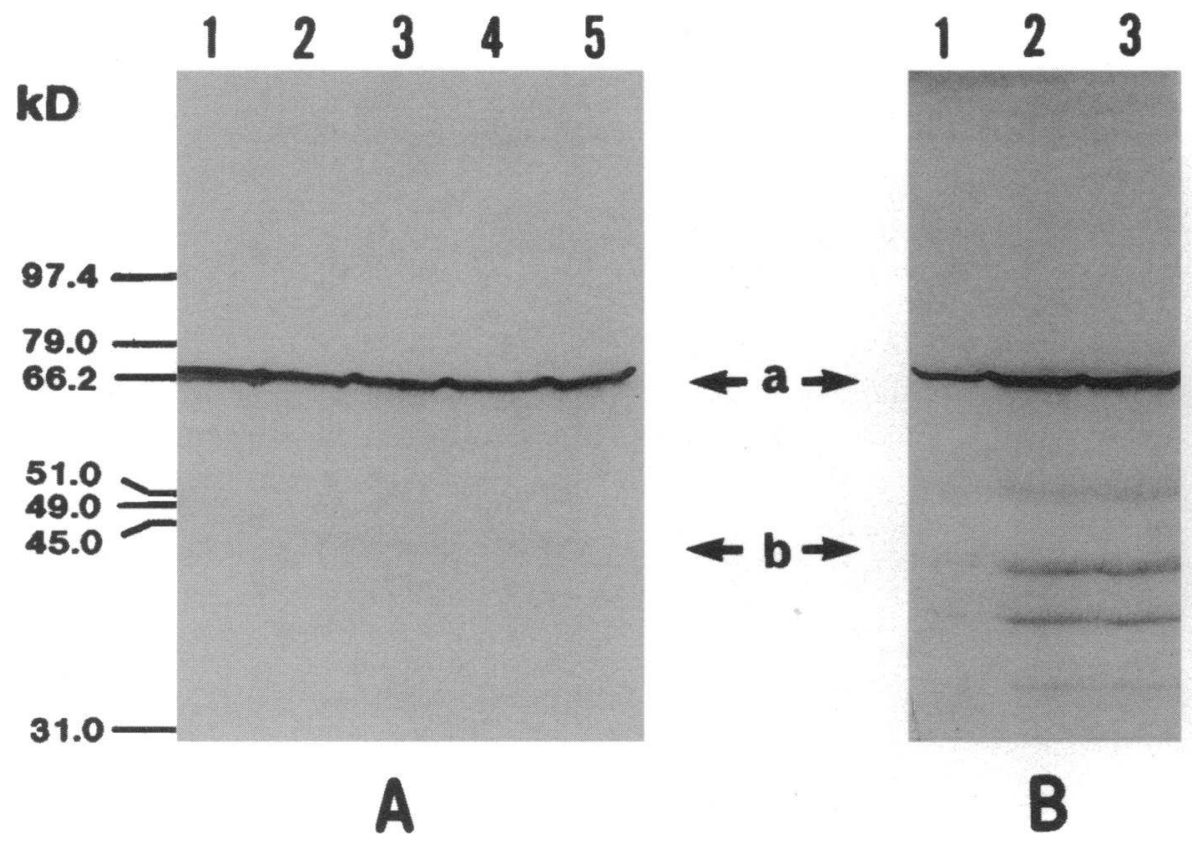

Figure 2. Immunoblot analysis of VLCAD protein in human livers and hearts. $50 \mu \mathrm{g}$ of liver or heart homogenate was electrophoresed on a $10 \%$ gel and transferred to nitrocellulose. The membrane was then developed using anti-human VLCAD antibody and alkaline phosphatase-conjugated second antibody. $A$, human livers. Lanes $1-5$, human liver samples $1-5$, respectively, described in Table II. B, human hearts. Lanes $1-3$, human heart samples 1-3, respectively, described in Table II. Arrows $a$ and $b$ indicate the positions of VLCAD and LCAD, respectively. of the band in the control fibroblasts. No band was observed at 6-, 24-, and 48-h chases in patient 1's fibroblasts even after the longer exposure $(168 \mathrm{~h})$. A faint fluorographic band was also detected only at the 1.5 -h pulse in patient 2 's fibroblasts (Fig. $4 \mathrm{~A}$ ). The band seemed to migrate at a slightly higher rate than the band in the control fibroblasts. No band was also observed at 6-, 24-, and 48-h chases in patient 2's fibroblasts after prolonged $(168 \mathrm{~h}$ ) fluorographic exposure. Since no band was detected at 6-, 24-, and 48-h chases in the fibroblasts from each of patients 3-7 even after the longer exposure (168 h), only the data concerning 1.5 -h pulse and competition experiment of 1.5-h pulse are summarized in Fig. $4 \mathrm{~B}$. A faint band was found at the $1.5-\mathrm{h}$ pulse in the fibroblasts from each of patients 3-7. Each band in patients 3 and 5 seemed to migrate at a slightly higher rate than the band in the control, as was observed in patient 2. The fluorographic bands for mitochondrial enoyl-CoA hydratase/3-hydroxyacyl-CoA dehydrogenase/3-ketoacyl-CoA thiolase trifunctional protein (35) were easily detectable both in the control and the seven patients' fibroblasts (data not shown). These results indicate that the seven patients' fibroblasts had a specific deficiency in the biosynthesis of VLCAD. A smaller VLCAD protein was detected only in the fibroblasts from patients 2,3 , and 5 , which suggests that these three patients may have a common mutation and that the natures of VLCAD-deficiency in the remaining four patients are heterogenous.

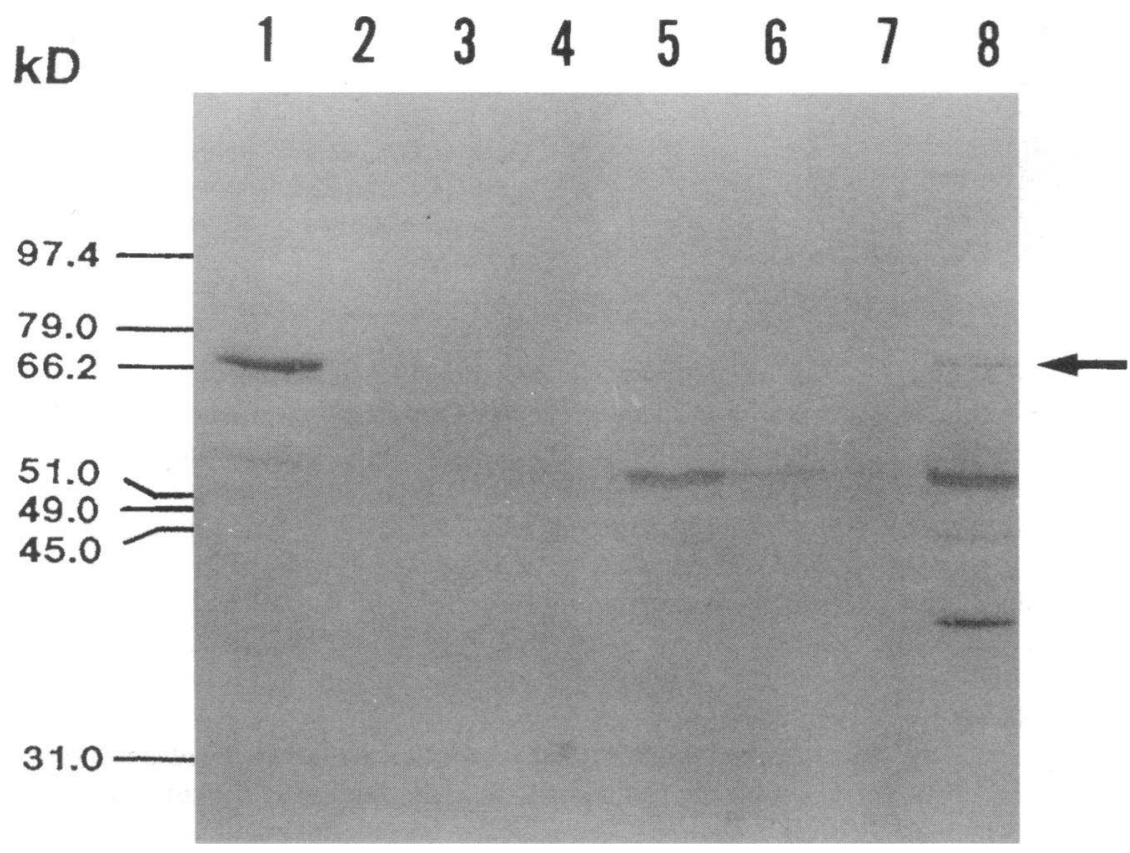

Figure 3. Immunoblot analysis of VLCAD protein in fibroblasts. $150 \mu \mathrm{g}$ of cell lysate was electrophoresed on a $10 \%$ gel and transferred to nitrocellulose. The membrane was then developed using anti-human VLCAD antibody and alkaline phosphatase-conjugated second antibody. Lane 1 , control; lane 2, patient 1 ; lane 3 , patient 2; lane 4 , patient 3 ; lane 5 , patient 4 ; lane 6 , patient 5 ; lane 7 , patient 6 ; and lane 8 , patient 7 . An arrow indicates the position of VLCAD. 

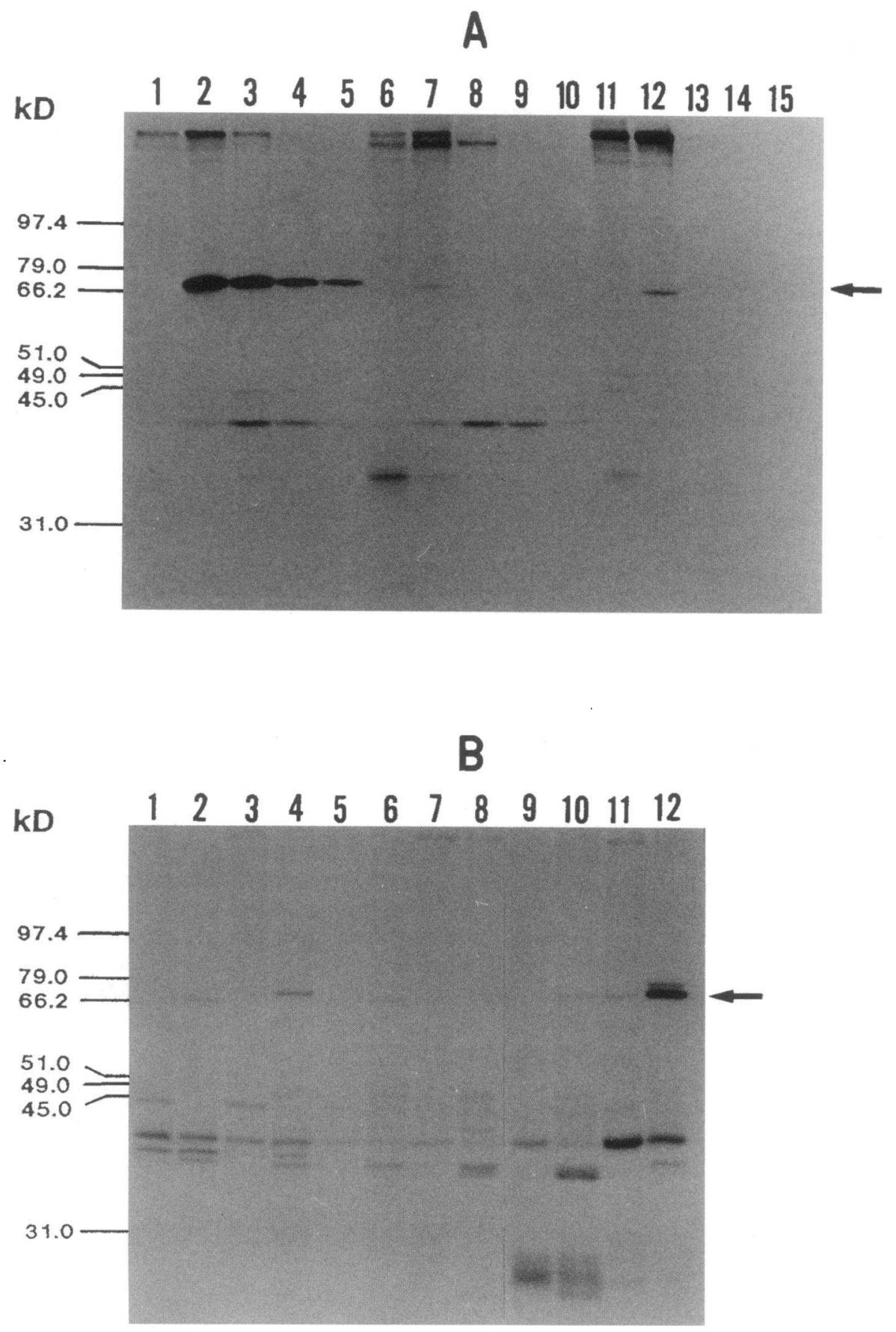

Figure 4. Pulse-labeling of VLCAD in fibroblasts. Pulse-chase experiments were conducted as described in Methods. X-ray film was exposed for $64 \mathrm{~h}$ at $-80^{\circ} \mathrm{C}$. A, pulselabeling and chase. Lanes 1-5, control; lanes $6-10$, patient 1; lanes $11-15$, patient 2.

Lanes 1,6 , and 11 , competition experiments of 1.5-h pulse; lanes 2, 7, and 12, 1.5-h pulse; lanes 3,8 , and $13,6-\mathrm{h}$ chase; lanes 4,9 , and 14, 24-h chase; lanes 5,10 , and 15, 48-h chase. $B$, pulse-labeling. Lanes 1 and 2 , patient 3; lanes 3 and 4, patient 4 ; lanes 5 and 6 , patient 5; lanes 7 and 8 , patient 6; lanes 9 and 10, patient 7; lanes 11 and 12 , control. Lanes (odd numbers), competition experiments of 1.5-h pulse; lanes (even numbers), 1.5-h pulse. An arrow indicates the position of VLCAD.

V LCAD-dependent palmitoyl-CoA dehydrogenation activity in patients' fibroblasts. To test whether the patients' fibroblasts exhibited VLCAD activity or not, palmitoyl-CoA dehydrogenation activity in patients' fibroblasts was measured in the presence and absence of anti-human VLCAD antibody. As shown in Table IV, the normal palmitoyl-CoA dehydrogenation activities (8.87-9.54 $\mathrm{mU} / \mathrm{mg}$ protein) found in the control fibroblasts were strongly reduced in the presence of anti-human VLCAD antibody. The data indicate that $>80 \%$ of the palmitoyl-CoA dehydrogenation in the control fibroblasts was catalyzed by VLCAD. The residual palmitoyl-CoA dehydrogenation activity (1.03-3.61 $\mathrm{mU} / \mathrm{mg}$ protein) found in the seven patients' fibroblasts was hardly inhibited in the presence of anti-human
VLCAD antibody, clearly indicating the absence of activity due to VLCAD in the patients' fibroblasts. Normal octanoyl-CoA dehydrogenation activities were found both in the control and the patients' fibroblasts (Table IV), indicating that the patients' fibroblasts had normal levels of MCAD. Since normal palmitoyl-CoA dehydrogenation activities $(7.64-10.31 \mathrm{mU} / \mathrm{mg}$ protein) were detected in the fibroblasts from the other 19 candidates, the possibility for a type of VLCAD deficiency having a normal level of the inactive variant VLCAD protein was excluded.

Long-chain fatty acid oxidation activity in fibroblasts. Table $\mathrm{V}$ shows the results of long-chain fatty acid $\beta$-oxidation in the control and the seven patients' fibroblasts. Long-chain fatty acid 
Table IV. Acyl-CoA Dehydrogenase Activity in Skin Fibroblasts

\begin{tabular}{cccccc}
\hline & \multicolumn{2}{c}{ Palmitoyl-CoA dehydrogenation $\left(\mathrm{C}_{16}\right)$} & $\begin{array}{c}\text { Octanoyl-CoA } \\
\text { dehydrogenation } \\
\left(\mathrm{C}_{8}\right)\end{array}$ & $\begin{array}{c}\text { Ratio of } \\
\mathrm{C}_{16} / \mathrm{C}_{\mathbf{8}}\end{array}$ \\
\cline { 2 - 4 } Sample & -Antibody & +Antibody & Ratio $+/-$ & & \\
\hline Patient & & & & & \\
1 & $1.80 \pm 0.19$ & $1.93 \pm 0.27$ & 1.07 & $4.26 \pm 0.21$ & 0.42 \\
2 & $1.03 \pm 0.25$ & $1.14 \pm 0.31$ & 1.11 & $3.45 \pm 0.35$ & 0.30 \\
3 & $1.95 \pm 0.35$ & $2.23 \pm 0.12$ & 1.14 & $3.65 \pm 0.27$ & 0.53 \\
4 & $3.61 \pm 0.27$ & $3.31 \pm 0.68$ & 0.92 & $5.22 \pm 0.31$ & 0.69 \\
5 & $1.66 \pm 0.43$ & $2.07 \pm 0.42$ & 1.25 & $2.93 \pm 0.08$ & 0.57 \\
6 & $2.98 \pm 0.77$ & $2.83 \pm 0.46$ & 0.95 & $6.83 \pm 0.24$ & 0.44 \\
7 & $1.56 \pm 0.13$ & $1.33 \pm 0.29$ & 0.85 & $3.11 \pm 0.30$ & 0.50 \\
Control & & & & & \\
1 & $8.87 \pm 0.29$ & $1.70 \pm 0.18$ & 0.19 & $2.87 \pm 0.12$ & 3.09 \\
2 & $9.54 \pm 0.52$ & $2.09 \pm 0.09$ & 0.22 & $3.04 \pm 0.33$ & 3.14 \\
3 & $9.13 \pm 0.93$ & $1.16 \pm 0.21$ & 0.13 & $3.38 \pm 0.22$ & 2.70 \\
4 & $9.02 \pm 0.86$ & $1.83 \pm 0.14$ & 0.20 & $3.12 \pm 0.30$ & 2.89 \\
& & & & & \\
\hline
\end{tabular}

Fibroblasts were treated as described in Table II. Activity unit is milliUnits per milligram of protein. Values are means $\pm \operatorname{SD}(n=3)$.

oxidation is known to be catalyzed both by mitochondrial and peroxisomal enzymes. POCA, a potent inhibitor of mitochondrial fatty acid $\beta$-oxidation system, was used to distinguish the mitochondrial and peroxisomal $\beta$-oxidation activities. In Table $\mathrm{V}$, the activity found in the absence of POCA (-POCA) represents the combined mitochondrial and peroxisomal activities, while that found in the presence of POCA ( +POCA) represents that in peroxisomes alone. In the control fibroblasts, $\sim 85 \%$ of palmitic acid oxidation was catalyzed by mitochondrial enzymes. The $\beta$-oxidation activities catalyzed by mitochondrial enzymes in the seven VLCAD-deficient fibroblasts were only $7-31 \%$ of those in the control fibroblasts. The $\beta$-oxidation activities catalyzed by peroxisomal enzymes in the seven patients' fibroblasts were similar to those in the control fibroblasts. These results demonstrate that the seven patients' fibroblasts had deficient long-chain fatty acid $\beta$-oxidation activity in mitochondria, but not in peroxisomes.

\section{Discussion}

We purified mitochondrial VLCAD from normal human liver and biochemically characterized it. We used the purified human enzyme as a standard for characterizing variant VLCAD in patients' cells, as well as an antigen to raise antibody. Normal human VLCAD exhibited activity toward palmitoyl-CoA, but not toward octanoyl-CoA or butyryl-CoA. Our results indicate that VLCAD catalyzes the major part of mitochondrial palmitoyl-CoA dehydrogenation in liver, heart, skeletal muscle, and skin fibroblasts. The data presented in this paper, together with those previously published $(21,29)$, suggest that human VLCAD is a rate-limiting enyzme in the long-chain fatty acid $\beta$-oxidation system and that VLCAD plays a central role in the catabolism of long-chain fatty acids in human tissues. This is in contrast to the previous prevailing view that such a central, rate-limiting role in the long-chain fatty acid $\beta$-oxidation was played by LCAD. In view of the new data on VLCAD and with reconsideration of the substrate specificity of rat LCAD (34, 36 ), it is likely that the role of LCAD is probably limited to the oxidation of $\mathrm{C}_{10}-\mathrm{C}_{14}$ acyl-CoAs.
Table V. Palmitic Acid $\beta$-Oxidation in Skin Fibroblasts

\begin{tabular}{cccccc}
\hline & \multicolumn{2}{c}{$\beta$-Oxidation activity } & & \multicolumn{2}{c}{ Mitochrondrial $\beta$-oxidation } \\
\cline { 2 - 3 } \cline { 5 - 6 } Fibroblast & -POCA & +POCA & & Activity* & Ratio of patientcontrols \\
\hline Patient & & & & \\
1 & $204 \pm 21$ & $109 \pm 14$ & & 95 & 0.13 \\
2 & $139 \pm 17$ & $86 \pm 8$ & & 53 & 0.07 \\
3 & $197 \pm 14$ & $121 \pm 19$ & 76 & 0.11 \\
4 & $312 \pm 19$ & $149 \pm 24$ & & 163 & 0.23 \\
5 & $156 \pm 16$ & $74 \pm 12$ & & 82 & 0.11 \\
6 & $143 \pm 10$ & $70 \pm 9$ & & 73 & 0.10 \\
7 & $367 \pm 29$ & $145 \pm 30$ & & 222 & 0.31 \\
Control & & & & - \\
1 & $895 \pm 49$ & $157 \pm 18$ & 738 & - \\
2 & $785 \pm 41$ & $131 \pm 20$ & 654 & - \\
3 & $840 \pm 53$ & $125 \pm 13$ & 715 & - \\
4 & $865 \pm 40$ & $106 \pm 19$ & 759 & \\
\hline
\end{tabular}

Values are means \pm SD $(n=3)$. * The value (-POCA) minus that of (+POCA). ${ }^{\ddagger}$ Ratio of patient activity versus mean of four control activities.

We found that the antibody raised against the purified human VLCAD was superior to that raised against purified rat VLCAD for the study of normal and variant VLCAD in human tissues. We recognized that, with the use of anti-human VLCAD antibody, an intense VLCAD band was detectable in the immunoblot analysis of human fibroblasts extracts, while no significant interfering band was observed. This is in contrast to the previous experience using antibody raised against rat VLCAD protein. With the use of anti-rat VLCAD antibody, the intensity of the cross-reactivity against the human VLCAD protein was only $25 \%$ of that observed with anti-human VLCAD antibody (Aoyama, T., and T. Hashimoto, unpublished observations). Furthermore, a strong nonspecific band near the VLCAD protein was observed when anti-rat VLCAD antibody was used (Aoyama, T., and T. Hashimoto, unpublished observations). Therefore, we consider that it is important to use anti-human VLCAD antibody in the analysis of human variant VLCAD.

The size of the VLCAD protein detected in human liver, heart, and skin fibroblast, respectively, was identical to that of the purified VLCAD protein. This is consistent with the previous data that the size and content of both the VLCAD protein and mRNA in various rat organs were identical, suggesting the existence of a single species of VLCAD in various organs (29).

In each of liver and heart samples, palmitoyl-CoA dehydrogenation activity measured by the method using 2,6-dichlorophenolindophenol and phenazine methosulfate (30) was roughly proportional to the amount of the VLCAD protein detected in immunoblot analysis (Table III, Fig. 2). In the study of fibroblasts, the assay method using 2,6-dichlorophenolindophenol and phenazine methosulfate was subject to interference and was unreliable. Therefore, the ferricenium method (31) was applied. Since the latter method is sensitive and reproducible, without being subject to interference, it is a useful and reliable method for the study of long-chain acyl-CoA dehydrogenase deficiencies using fibroblasts and lymphocytes.

The fibroblasts from patients 1-7 hardly contained the VLCAD protein (Fig. 3 ). These cells had a markedly reduced 
ability to synthesize the VLCAD protein (Fig. 4) and exhibited a low dehydrogenation activity toward palmitoyl-CoA (Table IV). These data indicate that all seven patients had VLCAD deficiency. Mitochondrial long-chain fatty acid oxidation activities in the seven VLCAD-deficient fibroblasts were only 7-31\% of the mean of the control fibroblasts (Table V). In patients with VLCAD deficiency, the impaired ability of the cells to oxidize fatty acids is probably responsible for the common symptoms, such as hypoketotic hypoglycemia, increased levels of longchain acylcarnitine esters, low levels of total serum carnitine, organic aciduria, hyperammonemia, hepatocellular dysfunction, and severe lipid storage in organs.

The results obtained in the immunoblot analysis (Fig. 3) and pulse-chase experiments (Fig. 4) suggest that the nature of the variant VLCAD protein in the seven patients is heterogenous. Since it is difficult to further define the nature of the deficiency at the protein level, mutation analysis at the DNA level needs to be carried out for better understanding of the cause of deficiency.

In this study, we analyzed human skin fibroblasts using antihuman VLCAD antibody and characterized VLCAD deficiency in detail. Bertand et al. (37) reported another method to identify VLCAD deficiency. They separated VLCAD and LCAD by differential solubilization of proteins in fibroblasts. We investigated this method by immunoblotting using anti-human VLCAD antibody. About 20 and $80 \%$ of VLCAD protein were distributed to the supernatant and membrane fractions, respectively, suggesting that it is possible to identify VLCAD deficiency by measuring palmitoyl-CoA dehydrogenation activity in membrane fraction; in contrast, it may be hard to identify isolated LCAD deficiency by measuring this activity in supernatant fraction containing significant amounts of contaminating VLCAD.

The clinical survey of the eight patients indicates that VLCAD deficiency appears to be widely distributed geographically. The sources of patients included in this study are as follows: six from North America (patients 1-6) and one each from Asia (patient 7) and Europe (37). The ethnic origins of the patients are seven Caucasians (patients 1-6) (37) and one Asian (patient 7).

The clinical features of VLCAD deficiency include early onset (within 4 mo after birth), high mortality (75\% within 2 mo after onset), and high frequencies of having liver dysfunction and cardiac disease ( $100 \%$, respectively). Among these, cardiac disease seems to be particularly important, because at least four patients presented with hypertrophic cardiomyopathy. This frequency $(>57 \%$ ) was rather high among diseases involving mitochondrial fatty acid oxidation and carnitine transport (38-42). The impaired long-chain fatty acid $\beta$-oxidation activity in the patients would cause the shortage of energy owing to the inadequate muscle fat utilization and the deficient production of ketone bodies, normally the preferential fuel for myocardium and skeletal muscle. It would also cause accumulation of long-chain triglycerides and long-chain acylcarnitines, which may be toxic to cardiac muscle (43). These unfavorable factors may relate to the occurrence of cardiac diseases such as arrhythmia, cardiomegaly, and cardiomyopathy, and muscular disorders such as episodic muscle pain, rhabdomyolysis, and progressive muscular weakness.

\section{Acknowledgments}

We thank Dr. H. Fukushima and Dr. N. Ito at Shinshu University School of Medicine for providing human livers and hearts and Ms. K. Yamada for preparation of the manuscript.
This study was supported by Grant-in Aid for Scientific Research (A) and Grant-in-Aid for Scientific Research on Priority Areas from the Ministry of Education, Science, and Culture of Japan, and a Research Grant for the Intractable Diseases from the Ministry of Health and Welfare of Japan.

\section{References}

1. Engel, P. C. 1992. Acyl-coenzyme A dehydrogenases. In Chemistry and Biochemistry of Flavoenzymes. F. Muller, editor. CRC Press, Boca Raton, FL. 597-655.

2. Rhead, W. J. 1991. Inborn errors of fatty acid oxidation in man. Clin. Biochem. 24:319-329.

3. Hale, D. E., and M. J. Bennett. 1992. Fatty acid oxidation disorders: a new class of metabolic diseases. J. Pediatr. 121:1-11.

4. Coates, P. M., and K. Tanaka. 1992. Molecular basis of mitochondrial fatty acid oxidation defects. J. Lipid Res. 33:1099-1110.

5. Kelly, D. P., A. J. Whelan, M. L. Ogden, R. Alpers, Z. Zhang, G. Bellus, N. Gregersen, I. Dorland, and A. W. Strauss. 1990. Molecular characterization of inherited medium-chain acyl-CoA dehydrogenase deficiency. Proc. Natl. Acad. Sci. USA. 87:9236-9240.

6. Yokota, I., Y. Indo, P. M. Coates, and K. Tanaka. 1990. Molecular basis of medium-chain acyl-coenzyme A dehydrogenase deficiency. J. Clin. Invest. 86:1000-1003

7. Kolvraa, S., N. Gregersen, E. Christensen, and N. Hobolth. 1982. In vitro fibroblast studies in a patient with $\mathrm{C} 6-\mathrm{C} 10$ dicarboxylic aciduria: evidence for a defect in general acyl-CoA dehydrogenase. Clin. Chim. Acta. 126:53-67.

8. Rhead, W. J., B. A. Amendt, K. S. Fritchman, and S. J. Felts. 1983. Dicarboxylic aciduria: deficient $\left[1-{ }^{14} \mathrm{C}\right]$ octanoate oxidation and medium-chain acyl-CoA dehydrogenase in fibroblasts. Science (Wash. DC). 221:73-75.

9. Stanley, C. A., D. E. Hale, P. M. Coates, C. L. Hall, B. E. Corkey, W. Yang, R. I. Kelley, E. L. Gonzalez, J. R. Williamson, and L. Baker. 1983. Mediumchain acyl-CoA dehydrogenase deficiency in children with non-ketotic hypoglycemia and low carnitine levels. Pediatr. Res. 17:877-884.

10. Yokota, I., P. M. Coates, D. E. Hale, P. Rinaldo, and K. Tanaka. 1991. Molecular survey of a prevalent mutation, ${ }^{985} \mathrm{~A}-$ to-G transition, and identification of five infrequent mutations in the medium-chain acyl-CoA dehydrogenase (MCAD) gene in 55 patients with MCAD deficiency. Am. J. Hum. Genet. 49:1280-1291.

11. Workshop on Molecular Aspects of MCAD Deficiency. 1992. Mutations causing medium-chain acyl-CoA dehydrogenase deficiency: a collaborative compilation of the data from 172 patients. In New Developments in Fatty Acid Oxidation. P. M. Coates and K. Tanaka, editors. Wiley-Liss, New York. 449456

12. Hale, D. E., M. Batshaw, P. M. Coates, F. E. Frerman, S. I. Goodman, I. Singh, and C. A. Stanley. 1985. Long-chain acyl-coenzyme A dehydrogenase deficiency: an inherited cause of non-ketotic hypoglycemia. Pediatr. Res. 19:666671.

13. Indo, Y., P. M. Coates, D. E. Hale, and K. Tanaka. 1991. Immunochemical characterization of variant long-chain acyl-CoA dehydrogenase in cultured fibroblasts from nine patients with long-chain acyl-CoA dehydrogenase deficiency. Pediatr. Res. 30:211-215.

14. Amendt, B. A., C. Greene, L. Sweetman, J. Cloherty, V. Shin, A. Moon, L. Teel, and W. J. Rhead. 1987. Short-chain acyl-CoA dehydrogenase deficiency: clinical and biochemical studies in two patients. J. Clin. Invest. 79:1303-1309.

15. Turnbull, D. M., K. Bartlett, D. L. Stevens, K. G. M. M. Albert, G. J. Gibson, M. A. Johnson, A. J. McCulloch, and H. S. A. Sherratt. 1984. Short-chain acyl-CoA dehydrogenase deficiency associated with a lipid-storage myopathy and secondary carnitine deficiency. N. Engl. J. Med. 311:1232-1236.

16. Coates, P. M., D. E. Hale, G. Finocchiaro, K. Tanaka, and S. M. Winter. 1988. Genetic deficiency of short-chain acyl-coenzyme A dehydrogenase in cultured fibroblasts from a patient with muscle carnitine deficiency and severe skeletal muscle weakness. J. Clin. Invest. 81:171-175.

17. Natio, E., Y. Indo, and K. Tanaka. 1990. Identification of two variant short-chain acyl-coenzyme A dehydrogenase alleles, each containing a different point mutation in a patient with short-chain acyl-coenzyme A dehydrogenase deficiency. J. Clin. Invest. 85:1575-1582.

18. Naito, E., Y. Indo, and K. Tanaka. 1989. Short-chain acyl-coenzyme A dehydrogenase (SCAD) deficiency: immunochemical demonstration of molecular heterogeneity due to variant SCAD with differing stability. J. Clin. Invest. 84:1671-1674.

19. Howat, A. J., M. J. Bennett, S. Variend, L. Shaw, and P. C. Engel. 1985 Defects in the metabolism of fatty acids in the sudden infant death syndrome. $B r$. Med. J. 290:1771-1775.

20. Allison, F., M. J. Bennett, S. Variend, and P. C. Engel. 1988. Acylcoenzyme A dehydrogenase deficiency in heart tissue from infants who died unexpectedly with fatty change in the liver. Br. Med. J. 296:11-20.

21. Izai, K., Y. Uchida, T. Orii, S. Yamatmoto, and T. Hashimoto. 1992. 
Novel fatty acid $\beta$-oxidation enzymes in rat liver mitochondria. I. Purification and properties of very-long-chain acyl-coenzyme A dehydrogenase. J. Biol. Chem. 267:1027-1033.

22. Aoyama, T., Y. Uchida, R. I. Kelley, M. Marble, K. Hofman, J. H. Tonsgard, W. J. Rhead, and T. Hashimoto. 1993. A novel disease with deficiency of mitochondrial very-long-chain acyl-CoA dehydrogenase. Biochem. Biophys. Res. Commun. 191:1369-1372.

23. Yamaguchi, S., Y. Indo, P. M. Coates, T. Hashimoto, and K. Tanaka. 1993. Identification of very-long-chain acyl-CoA dehydrogenase deficiency in three patients previously diagnosed with long-chain acyl-CoA dehydrogenase deficiency. Pediatr. Res. 34:111-113.

24. Tonsgard, J. H., J. K. Stephens, W. J. Rhead, D. Penn, A. L. Horwitz, B. S. Kirschner, P. F. Whitington, S. Berger, and M. E. Tripp. 1991. Defect in fatty acid oxidation: laboratory and pathologic findings in a patient. Pediatr. Neurol. 7:125-130.

25. Hale, D. E., C. A. Stanley, and P. M. Coates. 1990. The long-chain acylCoA dehydrogenase deficiency. In Fatty Acid Oxidation: Clinical, Biochemical and Molecular Aspects. K. Tanaka and P. M. Coates, editors. Alan R. Liss Inc., New York. 303-311.

26. Hale, D. E., C. A. Stanley, and P. M. Coates. 1990. Genetic defects of acyl-CoA dehydrogenases: studies using an electron transfer fiavoprotein reduction assay. In Fatty Acid Oxidation: Clinical, Biochemical and Molecular Aspects. K. Tanaka and P. M. Coates, editors. Alan R. Liss Inc., New York. 333-348.

27. Towbin, H., T. Staehelin, and J. Gordon. 1979. Electrophoretic transfer of proteins from acrylamide gels to nitrocellulose sheet. Proc. Natl. Acad. Sci. USA. 76:4350-4354.

28. Aoyama, T., M. Souri, T. Kamijo, S. Ushikubo, and T. Hashimoto. 1994. Peroxisomal acyl-coenzyme A oxidase is a rate-limiting enzyme in a very-longchain fatty acid $\beta$-oxidation system. Biochem. Biophys. Res. Commun. 201:15411547.

29. Aoyama, T., I. Ueno, T. Kamijo, and T. Hashimoto. 1994. Rat very-longchain acyl-CoA dehydrogenase, a novel mitochondrial acyl-CoA dehydrogenase gene product, is a rate-limiting enzyme in long-chain fatty acid $\beta$-oxidation system: cDNA and deduced amino acid sequence and distinct specificities of the cDNA-expressed protein. J. Biol. Chem. 269:19088-19094.

30. Furuta, S., S. Miyazawa, and T. Hashimoto. 1981. Purification and properties of rat liver acyl-CoA dehydrogenases and electron transfer flavoprotein. $J$. Biochem. 90:1739-1750.

31. Lehman, T. C., D. E. Hale, A. Bhala, and C. Thorpe. 1990. An acylcoenzyme A dehydrogenase assay utilizing the ferricenium ion. Anal. Biochem. 186:280-284.
32. Massey, V. 1959. The microestimation of succinate and the extinction coefficient of cytochrome $c$. Biochim. Biophys. Acta. 34:255-256.

33. Hashimoto, T. 1992. Peroxisomal and mitochondrial enzymes. In New Developments in Fatty Acid Oxidation. P. M. Coates and K. Tanaka, editors. Wiley-Liss, Inc., New York. 19-32.

34. Ikeda, Y., K. Okamura-Ikeda, and K. Tanaka. 1985. Purification and characterization of short-chain, medium-chain, and long-chain acyl-CoA dehydrogenases from rat liver mitochondria: isolation of the holo- and apoenzymes and conversion of the apoenzyme to the holoenzyme. $J$. Biol. Chem. 260:1311-1325.

35. Kamijo, T., R. J. A. Wanders, J. M. Saudubray, T. Aoyama, A. Komiyama, and T. Hashimoto. 1994. Mitochondrial trifunctional protein deficiency: catalytic heterogeneity of the mutant enzyme in two patients. J. Clin. Invest. 93:17401747.

36. Ikeda, Y., C. Dabrowski, and K. Tanaka. 1983. Separation and properties of five distinct acyl-CoA dehydrogenases from rat liver mitochondria: identification of a new 2-methyl branched chain acyl-CoA dehydrogenase. J. Biol. Chem. 258:1066-1076.

37. Bertand, C., C. Largilliere, M. T. Zabot, M. Mathieu, and C. VianeySaban. 1993. Very long chain acyl-CoA dehydrogenase deificiency: identification of a new inborn error of mitochondrial fatty acid oxidation in fibroblasts. Biochem. Biophys. Acta. 1180:327-329.

38. Tripp, M. E., M. L. Katcher, H. A. Peters, E. F. Gilbert, S. Arya, R. J. Hodach, and A. L. Shug. 1981. Systemic carnitine deficiency presenting as endocardial fibroelastosis: a treatable cardiomyopathy. N. Engl. J. Med. 305:385-390.

39. Stanley, C. A., S. DeLeeuw, P. M. Coates, C. Vianey-Liaud, P. Divry, J. P. Bonnefont, J. M. Saudubray, M. Haymond, F. K. Trefz, G. N. Bremingstall, et al. 1991. Chronic cardiomyopathy and weakness or acute coma in children with a defect in carnitine uptake. Ann. Neurol. 30:700-716.

40. Saudubray, J. M., G. Mitchell, J. P. Bonnefont, G. Schwartz, C. Nuttin, A. Munnich, M. Brivet, A. Vassault, F. Demaugre, D. Rabier, and C. Charpentier. 1992. Approach to the patient with a fatty acid oxidation disorder. In New Developments in Fatty Acid Oxidation. P. M. Coates and K. Tanaka, editors. WileyLiss, Inc., New York. 271-288.

41. Treem, W. R., C. A. Stanley, D. N. Finegold, D. E. Hale, and P. M Coates. 1988. Primary carnitine deficiency due to a failure of carnitine transport in kidney, muscle, and fibroblasts. N. Engl. J. Med. 319:1331-1336.

42. Demaugre, F., J. P. Bonnefont, M. Colonna, C. Cepanec, J. P. Leroux, and J. M. Saudubray. 1991. Infantile form of carnitine palmitoyltransferase II deficiency with hepatomuscular symptoms and sudden death. J. Clin. Invest. 87:859-864.

43. Corr, P. B., M. H. Creer, K. A. Yamada, J. E. Saffitz, and B. E. Sobel. 1989. Prophylaxis of early ventricular fibrillation by inhibition of acylcarnitine accumulation. J. Clin. Invest. 83:927-936. 